\title{
Collaborating with the Enemy: Function of Macrophages in the Development of Neoplastic Disease
}

\author{
Andrzej Eljaszewicz, ${ }^{1}$ Malgorzata Wiese, ${ }^{1}$ Anna Helmin-Basa, ${ }^{1}$ \\ Michal Jankowski, ${ }^{2,3}$ Lidia Gackowska, ${ }^{1}$ Izabela Kubiszewska, ${ }^{1}$ Wojciech Kaszewski, ${ }^{1}$ \\ Jacek Michalkiewicz, ${ }^{1,4}$ and Wojciech Zegarski ${ }^{2,3}$ \\ ${ }^{1}$ Chair of Immunology, Collegium Medicum in Bydgoszcz, Nicolaus Copernicus University of Torun, \\ ul. M. Sklodowskiej-Curie 9, 85-094 Bydgoszcz, Poland \\ ${ }^{2}$ Department of Surgical Oncology, Collegium Medicum in Bydgoszcz, Nicolaus Copernicus University of Torun, \\ ul. Romanowskiej 2, 85-796 Bydgoszcz, Poland \\ ${ }^{3}$ Department of Surgical Oncology, Prof. F. Łukaszczyk Memorial Center of Oncology in Bydgoszcz, \\ ul. Romanowskiej 2, 85-796 Bydgoszcz, Poland \\ ${ }^{4}$ Department of Microbiology and Immunology, Children's Memorial Hospital, \\ ul. Aleja Dzieci Polskich 20, 04-001 Warsaw, Poland
}

Correspondence should be addressed to Andrzej Eljaszewicz; a.eljaszewicz@cm.umk.pl

Received 14 October 2012; Revised 26 December 2012; Accepted 13 January 2013

Academic Editor: Wei Sung-Jen

Copyright (C) 2013 Andrzej Eljaszewicz et al. This is an open access article distributed under the Creative Commons Attribution License, which permits unrestricted use, distribution, and reproduction in any medium, provided the original work is properly cited.

Due to the profile of released mediators (such as cytokines, chemokines, growth factors, etc.), neoplastic cells modulate the activity of immune system, directly affecting its components both locally and peripherally. This is reflected by the limited antineoplastic activity of the immune system (immunosuppressive effect), induction of tolerance to neoplastic antigens, and the promotion of processes associated with the proliferation of neoplastic tissue. Most of these responses are macrophages dependent, since these cells show proangiogenic properties, attenuate the adaptive response (anergization of naïve T lymphocytes, induction of Treg cell formation, polarization of immune response towards Th2, etc.), and support invasion and metastases formation. Tumorassociated macrophages (TAMs), a predominant component of leukocytic infiltrate, "cooperate" with the neoplastic tissue, leading to the intensified proliferation and the immune escape of the latter. This paper characterizes the function of macrophages in the development of neoplastic disease.

\section{Introduction}

Human body is exposed to a continuous influence of carcinogenic factors (physical, chemical, and biological), representing one of the reasons for the development of genetic mutations. Cells possess an array of mechanisms able to prevent mutations, as well as to repair DNA defects and eliminate genetically altered cells, for example, by the means of apoptosis [1,2]. Disorders of this complicated protective system lead to the development of neoplastic cells, which, in turn, may be eliminated by an array of immunological mechanisms, including those affected by the innate immune system (monocytes, macrophages, NK cells, cytokines, etc.) and the adaptive immunity (induction of $\mathrm{T}$ and $\mathrm{B}$ lymphocytes). In order to eliminate neoplastic cells, the cells of the immune system must recognize them as "foreign." The principles of recognition and the mechanisms of the immunological response are similar to those induced by foreign (bacterial, viral) or own antigens (autoantigens). Foreign antigens are highly immunogenic; that is, they induce immune response aimed at the rapid elimination of the infectious factors. These processes lead to the selection of a pool of immunocompetent cells, specialized in the destruction of a given factor. As previously mentioned, neoplastic cells originate from genetically altered cells of own tissues and therefore contain components that induce various degrees of immune tolerance, protecting 
them against the elimination by immunological mechanisms. On the other hand, neoplastic cells are characterized by a genetic instability [3], which manifests by changes in antigenic profile, which are not observed in the normal tissue. In some cases, this is accompanied by the overexpression of genes that remain inactive or exhibit low-level activity under normal conditions; many of the factors produced in this manner act as immunomodulators. It is widely known that neoplastic tissue can directly modulate its growth environment as a result of the activity of secreted cytokines and chemokines. This may be due to the following:

(a) chemotactic effect on leukocytes, including monocytes and macrophages;

(b) suppression of the activity of the immune system;

(c) regulation of neovascularization processes [4].

\section{Chemotactic Factors for Monocytes/Macrophages Released by Neoplastic Cells}

Some factors synthesized and released by neoplastic cells can induce leukocyte chemotaxis, including peripheral monocytes and macrophages located in the surrounding tissues. These cells represent the predominant component of leukocytic infiltrate of many tumors and, due to their pleotropic biological activity control, the majority of immunological processes proceeding in the region of neoplastic growth. Chemotaxis of monocytes and macrophages is a receptordependent process [5], directly associated with the polarization of these cells towards pro- or anti-inflammatory cells.

Monocyte chemotactic protein-1 (MCP-1/CCL2), showing affinity to CCR2 receptor, constitutes one of the principal monocyte/macrophage chemokines [6,7]. Moreover, the concentration of CCL2 correlates positively with the stage of the tumor in cancer of urinary bladder and breast. High level of this cytokine is associated with poor prognosis; it is observed in patients with higher clinical stages of the tumor $[8,9]$. In contrast, a proportional decrease in the concentration of CCL2 is observed with increasing stages of gastric malignancies, both in the serum and in neoplastic tissue. Perhaps, this phenomenon results from enhanced "consumption" of MCP-1 and concurrent unchanged level of synthesis [10]. Interestingly, MCP-1 can be released also by macrophages located in the region of neoplastic growth [11]. This suggests direct involvement of these cells in the recruitment of peripheral monocytes. It should be noted, however, that the impaired expression of CCR2 receptor on the surface of tumor-associated macrophages leads to the reversal of the effect exerted on these cells by the discussed chemokine. Plausibly, this is one of the mechanisms enabling the maintenance of rich macrophage infiltrate in the region of neoplastic growth [12]. Expression of MCP1 correlates positively with the level of vascular endothelial growth factor (VEGF), TNF- $\alpha$, and IL-8, which suggests its involvement in the processes of neovascularization [11]. The migration of peripheral monocytes to the site of neoplastic growth can be also induced by monocyte chemotactic protein-2 (MCP-2/CCL-8) and monocyte chemotactic protein-3 (MCP-3/CCL-7). These cytokines show both a structural similarity to MCP-1 and an affinity to the CCR2 receptor [13]. The chemotaxis of monocytes is also induced by CCL5 (RANTES), whose level of expression correlates with the degree of macrophage infiltration and lymph node metastases of neoplastic cells [14]. RANTES and CCL2 stimulate monocytes to secrete metalloproteinase-9 (MMP-9) and metalloproteinase-19 (MMP-19), which suggests indirect involvement of the discussed chemokines in the degradation of basal membrane, and hence in the process of neovascularization $[15,16]$. Furthermore, high concentration of CCL5 increases the probability of metastases in patients with gastric malignancies [17]. Also, factors released by the neoplastic cells, such as VEGF, IL-8, and angiopoietin-2 (Ang-2), exert chemotactic effect on monocytes/macrophages; additionally, they are involved in the processes of angiogenesis.

\section{Regulation of the Process of Neovascularization}

Angiogenesis, although physiologically necessary, underlies a number of diseases. Formation of new blood vessels is critical for neoplastic growth and results from the predominance of proangiogenic factors over those inhibiting angiogenesis [18]. Enhanced angiogenesis can be observed in very early stages of malignant growth $[19,20]$. Molecules such as (VEGF), interleukin-8 (IL-8/CXCL8), basic fibroblast growth factor (bFGF), angiopoietin-1 (Ang-1), and angiopoietin-2 (Ang-2) are the main mediators of neovascularization released by the neoplastic cells, including the cells of gastric malignancies.

VEGF is synthesized and secreted by many types of neoplasms [21-23] and although high levels of this molecule can be observed both in the plasma and in the serum of patients, the serum concentration of VEGF is higher. This results from the secretory activity of thrombocytes, which release high amounts of this factor during the coagulation of blood [17]. The production of VEGF in human macrophages is regulated by $\mathrm{NF} \kappa \mathrm{B}$ [24]. VEGF acts in the receptor-dependent manner [25], inducing the chemotaxis of peripheral monocytes as a result of interacting with VEGF-R1 [26]. In response to VEGF, activated monocytes/macrophages synthesize molecules, for example, metalloproteinase- 9 [27, 28], which, as previously mentioned, is involved in the processes of angiogenesis. Although tumor-associated macrophages (TAM's) constitute the principal source of MMP9 in the zone of neoplastic growth, it should be noted that this molecule may be also synthesized by neoplastic cells, stromal neutrophils, fibroblasts, and mastocytes [29]. Moreover, the activity of VEGF leads to an increased permeability of blood vessels within the neoplastic tissue [30], proliferation of vascular endothelial cells [31], and the inhibition of dendritic cell maturation [32]. The majority of these processes are associated with the activation of VEGF-R2 receptor [33]. Therefore, the VEGF/VEGF-R2 system is connected with the initiation of neovascularization processes.

Similarly to VEGF, interleukin-8 is a chemokine with proangiogenic activity. High expression levels of this molecule 
have been observed in various types of neoplasms, directly correlated with the vascularization of proliferating tissue and poor prognosis, being the highest in the advanced stages of tumor development. Gastric cancer cells also show the expression of A (CXCR1) and B (CXCR2) receptors for IL8. During in vitro IL-8 stimulation, they show increased expression of epidermal growth factor receptor (EGFR), MMP-9, VEGF, and IL-8 [34]. Similarly to VEGF, interleukin8 induces the migration of monocytes/macrophages to the site of neoplastic growth.

Basic fibroblast growth factor (bFGF) is one of the strongest stimulators of angiogenesis [35], acting via FGF$\mathrm{R} 1$ and FGF-R2 receptors. Its expression is observed in many types of neoplasms [36]. High level of bFGF correlates positively with poor prognosis, and its expression in neoplastic cells is associated with the vascularization of the tumor. Moreover, this factor stimulates the chemotaxis of macrophages [37], which acquire the potential to synthesize and secrete this molecule in response to mediators released by neoplastic cells [38]. This suggests indirect TAMs-dependent influence of bFGF on the processes of tumor neovascularization.

Angiopoetin-1 (Ang-1) and angiopoetin-2 (Ang-2) are the main representatives of angiopoietin family. The activity of Ang-2 during the development of neoplastic disease is associated with the progression of the disease and the neovascularization of the tumor [39]. Angiopoietin-1 activates Tie2 receptor (angiopoietin receptor), in this way stimulating in vitro migration of endothelial cells; moreover, it recruits pericytes into the newly formed vessels in order to stabilize their structure. In contrast, angiopoietin-2 is a natural antagonist of Tie-2 receptor. Ang- 2 inhibits the maturation of vessels resulting from Ang-1 activity in a VEGF-independent manner and causes their regression. Therefore, it plays a regulatory function [34]. The activity of Ang-2 leads to the destabilization of vessels, which is necessary for the initiation of neovascularization process [40]. Angiopoietin2 exerts positive effects on the processes of angiogenesis through VEGF involvement [34]. Interestingly, VEGF causes an increase in Ang-1 expression, but it does not modulate the synthesis of Ang-2 [41]. The level of Ang-2 expression correlates significantly with the clinical stage of disease (lymph node and organ metastases) while the expression of Ang-1 is significantly higher in poorly differentiated tumors [40]. The angiopoietin/Tie-2 system is involved in the remodeling and maturation of blood vessels and is, therefore, complementary to the activity of VEGF [34]. Moreover, the evaluation of Ang1, Ang-2, and Tie-2 serum concentrations seems to be useful in preoperative differentiation of malignant thyroid tumors [42]. Additionally, angiopoietins are able to stimulate the chemotaxis of Tie-2-positive peripheral monocytes, which, constituting cells with pro-angiogenic potential, support the proliferation of neoplastic tissue [43].

\section{Immunosuppressive Effect}

Cancer cells and tumor-infiltrating leukocytes (primarily macrophages) modulate the activity of the immune system, also by means of immunosuppression, via the profile of released factors (cytokines and chemokines). IL-10 and TGF$\beta$ are released by these cells and show an array of immunosuppressive effects, for example:

(a) blockade of the activity of cytotoxic NK cells [44], macrophages, and cytotoxic T lymphocytes $\left(\mathrm{CD} 8^{+}\right)$ [45];

(b) reduced expression of class II MHC molecule on the surface of antigen presenting cells [46];

(c) polarization of immune response towards Th2 [47];

(d) inhibition of dendritic cell maturation [48];

(e) inhibition of certain functions of T lymphocytes [49];

(f) stimulation of tumor cell B7-H3 expression [50].

Together with a strong anti-inflammatory signal, neoplastic cell-released chemotactic factors for monocytes and macrophages induce their differentiation into MII macrophages, and hence into cells showing an array of functions promoting the proliferation of neoplastic tissue. Consequently, it should be noted that aside from direct immunosuppressive activity, growing neoplasm induces antiinflammatory activity of infiltrating cells, thus escaping from the control of the immune system.

Macrophages are terminally differentiated cells of bone marrow origin that reside in tissues and are derived from peripheral monocytes (Figure 1). Depending on the activating factor, monocytes and macrophages can be involved in an array of biological processes, such as:

(a) presentation of antigen;

(b) cytotoxicity;

(c) phagocytosis;

(d) secretion of biologically active molecules;

(e) control of inflammatory processes;

(f) rearrangement and reconstruction of destroyed tissues [51].

As previously mentioned, the proliferation of neoplastic tissue modulates the activity of immune cells, including the function of monocytes and macrophages. It is widely known that macrophages residing at the site of neoplastic growth, referred to as TAMs, constitute the predominant component of infiltrate in many neoplasms, including gastric malignancies [52-54]. Due to their pleotropic biological properties, TAMs can have both progressive and regressive effects on the development of neoplastic tissue. Moreover, they control primary and secondary immune responses. The pro- or antineoplastic activity of macrophages is directly associated with their pro- or anti-inflammatory activity, respectively, and tightly depends upon monocyte-activating factors, which define the relevant polarization of these cells (Figure 1).

\section{Activity of Monocytes}

As peripheral cells, monocytes do not have direct contact with a neoplastic tissue. Indirectly, however, they are subject to its immunomodulatory effect, responding to chemotactic 


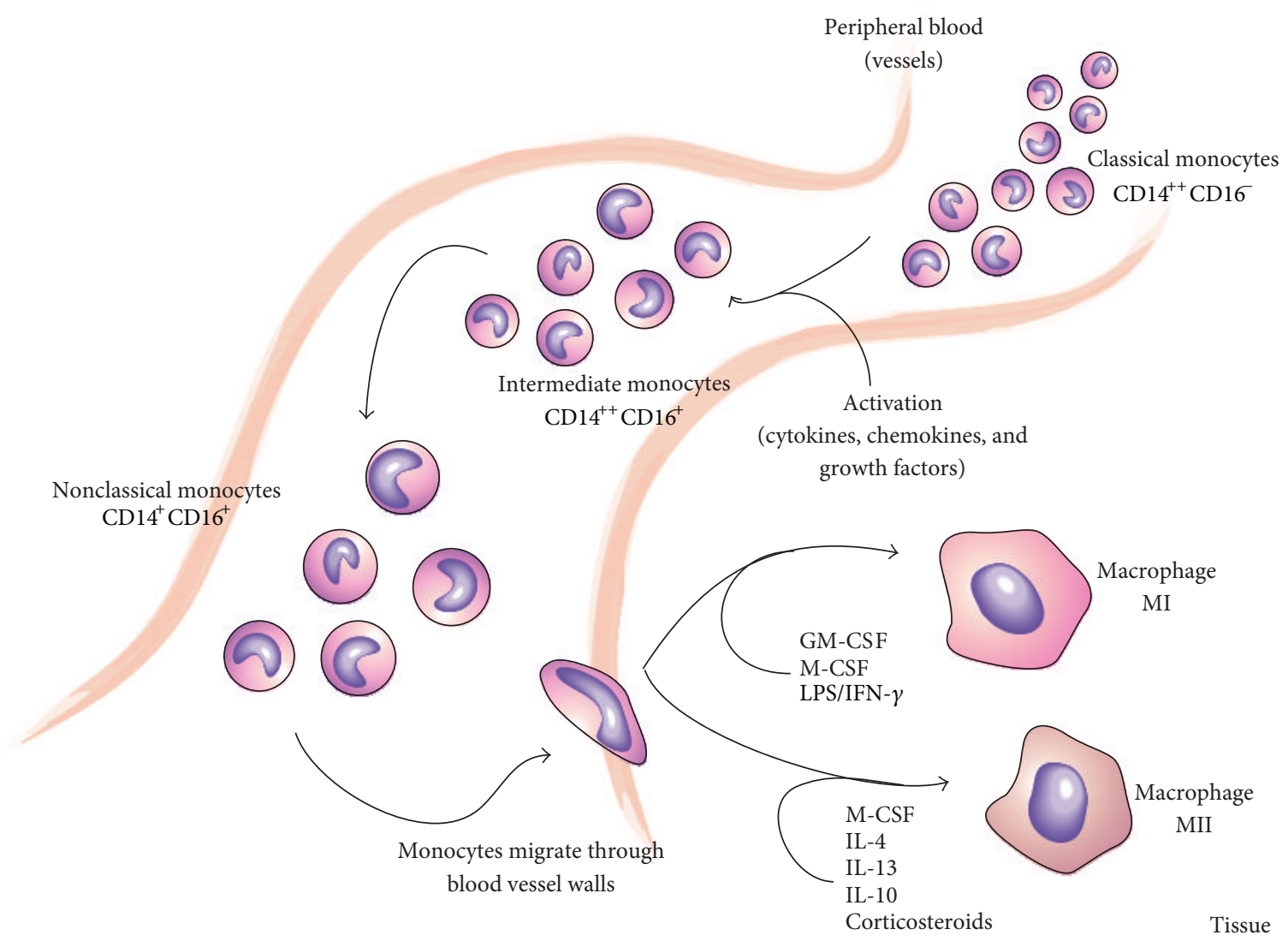

FIGURE 1: Differentiation of monocytes towards macrophages.

factors and neoplastic antigens present in peripheral blood, as well as to circulating neoplastic cells. All these factors directly induce the differentiation of monocytes to macrophages and their polarization towards pro- or anti-inflammatory cells. In the case of some malignancies, such as colorectal cancer, elevated monocyte count is considered as an independent prognostic factor [55].

Monocytes are heterogeneous population of cells in terms of morphology, phenotype, and effector properties. On the basis of the level of expression of lipopolysaccharide (LPS) receptor (CD14) and $\mathrm{Fc} \gamma$ receptor III (CD16), they can be classified into three well-characterized subpopulations

(a) those showing strong expression of CD14 receptor and lacking the expression of $\mathrm{CD} 16$ receptor $\left(\mathrm{CD} 14^{++} \mathrm{CD} 16^{-}\right)$;

(b) those showing strong expression of CD14 and the presence of $\mathrm{CD} 16$ receptor $\left(\mathrm{CD} 14^{++} \mathrm{CD} 16^{+}\right)$;

(c) those showing weaker expression of CD16 receptor than in the above-mentioned groups expression of $\mathrm{CD} 14$ receptor, with simultaneous $\left(\mathrm{CD} 14^{+} \mathrm{CD} 16^{+}\right)$ [56].

Monocytes with $\mathrm{CD} 14^{++} \mathrm{CD} 16^{-}$phenotype are referred to as classical monocytes and correspond to $85-95 \%$ of all peripheral monocytes under physiological conditions. The remaining two populations showing strong expression of
CD16 receptor differ from each other in terms of CD14 expression level and, under physiological conditions, represent up to $15 \%$ of peripheral monocytes $[57,58]$. Despite phenotypic similarities associated with the expression of CD16 receptor, stronger, as compared to classical monocytes, expression of HLA-DR, CD86, and CD54, and lower level of CD64 expression, both of the aforementioned subpopulations of monocytes show different biological activities [59]. An increase in the fraction of $\mathrm{CD} 14^{+} \mathrm{CD} 16^{+}$subpopulation of peripheral monocytes was observed during infections and inflammatory processes $[60,61]$, in septic states [62], and in some types of neoplasms $[63,64]$. $\mathrm{CD}^{+} 4^{+} \mathrm{CD} 16^{+}$ cells are referred to as proinflammatory monocytes, because in contrast to classical monocytes upon stimulation they synthesize and release high amounts of tumor necrosis factor-alpha (TNF- $\alpha$ ) without concomitant secretion of IL10 or with low secretion of this cytokine [65]. The cells from this population show an array of similarities to tissue macrophages, and they are, therefore, postulated to be more mature and macrophage-like cells than the classical monocytes [66, 67]. Higher fraction of monocytes from $\mathrm{CD} 14^{++} \mathrm{CD} 16^{+}$subpopulation has been observed in a number of conditions, including septic neonatal states [68] and gastric malignancies [69]. Additionally, compared to the proinflammatory subpopulation and classical monocytes, these cells show a higher expression of CD11b and TLR4 [59]. Moreover, they differ from the $\mathrm{CD} 14^{+} \mathrm{CD} 16^{+}$subpopulation 
in terms of characteristics such as higher phagocytic activity [70] and the presence of anti-inflammatory properties, constituting the principal source of IL-10 amongst peripheral monocytes. The presence of this subpopulation in peripheral blood is thought to constitute an intermediate stage in the differentiation of monocytes to macrophages [59].

In spite of the lack of direct contact with neoplastic tissue, peripheral monocytes represent an interesting object during the assessment of the developmental stage of the disease. As previously mentioned, they undergo a continuous stimulation by chemokines and cytokines released by neoplastic cells and tumor-infiltrating cells, as well as by neoplastic antigens and circulating neoplastic cells.

$\mathrm{CD} 14^{+} \mathrm{CD} 16^{+}$cells are the main subpopulation of monocytes showing in vitro antineoplastic activity, which is directly associated with the enhanced synthesis and secretion of cytokines such as TNF- $\alpha$, IL-12p40, and IL-12p70, lack of synthesis and release of IL-10, enhanced synthesis of reactive nitrogen species, and higher cytotoxic and cytostatic activities [71]. Obviously, IL-12p40 and IL-12p70 do not exert direct anti-neoplastic effect. The influence of IL-12p70 is associated with the activation of IFN- $\gamma$ synthesis in lymphocytes, which in turn contributes to the polarization of immune response towards Th1, that is, a proinflammatory response against neoplastic cells. Additionally, IL-12p70 activates cytotoxic $\mathrm{T}$ lymphocytes $\left(\mathrm{CD}^{+} \mathrm{CD}^{+}\right)$and $\mathrm{NK}$ cells, both showing an array of antineoplastic properties [72]. IL-12p40 is a chemotactic factor for monocytes, which differentiate into macrophages and migrate to the tissue. Therefore, IL-12p40 enhances the infiltration of macrophages into the site of neoplastic proliferation [73], where, as pro-inflammatory cells, they can exert many antineoplastic effects. The results of in vitro studies suggest that increased fraction of $\mathrm{CD}^{+} 6^{+}$monocytes in patients with malignancies can represent a natural consequence of immune response against neoplastic tissue as well as against circulating neoplastic cells. Spontaneous increase in this population of peripheral monocytes was also observed in vivo, including gastrointestinal malignancies [64].

Additionally, a decrease in the fraction of the subpopulation 1 of T-helper lymphocytes (Th1) was observed in peripheral blood of cancer patients in relation to the subpopulation 2 (Th2); this was associated with lower plasma concentration of molecules such as IL- 2 and IFN- $\gamma$, and higher level of cytokines, such as IL-4, IL-10, and IL-13, as compared to healthy individuals [74]. In view of the elevated concentration of anti-inflammatory factors, circulating monocytes should gain anti-inflammatory properties and differentiate into MII macrophages, which are involved in the processes of neovascularization, among others. Proangiogenic activity is also characteristic for monocytes that show the surface expression of angiopoietin receptor (Tie-2), although this receptor is mostly expressed on epithelial cells and is considered as a specific feature of vascular epithelial cells [75]. Tie- $2^{+}$ monocytes represent a separate population of cells referred to as the Tie-2-expressing monocytes (TEMs). Even though their physiological fraction in peripheral blood is low and corresponds to only 1-2\% of peripheral leukocytes, approximately $20 \%$ of circulating monocytes are Tie- $2^{+}[43,76]$.
An increase in the fraction of Tie-2-expressing monocytes, even up to $10 \%$ of all peripheral blood mononuclear cells (PBMCs), has been observed in cancer patients. Murine model studies revealed strong pro-angiogenic properties of TEMs during the processes of neoplastic tissue neovascularization. Moreover, these cells are considered the precursors of pro-angiogenic tissue macrophages [76], which correspond to up to $30 \%$ of all TAMs in certain parts of the neoplastic tissue [77].

\section{Macrophage Activity}

Macrophages are the predominant cells of the leukocytic infiltrate of many neoplasms and are able to polarize their immune response in both a pro- or anti-inflammatory direction. Monocytes, activated by microorganisms or their parts, certain pro-inflammatory cytokines (e.g., IFN- $\gamma$ ), GM-CSF, and M-CSF, migrate into tissues and differentiate into proinflammatory cells, referred to as MI macrophages, which are involved in the destruction of microorganisms and neoplastic cells, among others (Table 1). Their function includes the activation of immune system and the support of adaptive response by means of:

(a) enhanced synthesis and secretion of pro-inflammatory cytokines such as TNF- $\alpha$, IL-1, IL-6, IL-12, and IL-23 [78-80];

(b) enhanced synthesis and secretion of chemokines such as CCL5, CCL8, CXCL2, and CXCL4 [81-83];

(c) polarization of immune response towards Th1 and/or Th17 [84];

(d) high capacity for presentation of antigen to antigennaive T lymphocytes;

(e) cytotoxic potential [85].

Monocytes activated by factors such as IL-4, IL-13, IL10 , and M-CSF show typical activities of anti-inflammatory cells; when present in tissues, they are referred to as MII macrophages (Table 1). These cells are characterized by:

(a) enhanced synthesis and secretion of anti-inflammatory cytokines such as IL-10, TGF- $\beta$, and IL-1RA;

(b) enhanced synthesis and secretion of chemokines such as CCL16, CCL18, and CCL22;

(c) polarization of immune response towards Th2;

(d) induction of T-regulatory (Treg) lymphocyte formation;

(e) low capacity for the presentation of antigen to antigen-naive T lymphocytes;

(f) strong expression of arginase-1 (its activity alters the metabolism of L-arginine into ornithine and polyamines, which results in the blockade of inducible nitric oxide synthase (iNOS));

(g) lack of cytotoxic activity;

(h) higher expression of certain membrane receptors, including type $2 \mathrm{Fc}$ receptor for $\operatorname{IgG}$ (Fc-R2, CD23), 
TABLE 1: Macrophage subsets.

\begin{tabular}{lll}
\hline Populations & Inducing agents & Functions \\
\hline & & (i) High capacity for antigen presentation \\
MI & GM-CSF; & (ii) Thl polarization \\
& IFN- $\gamma+$ LPS; & (iii) Defense against bacteria \\
& TNF- $\alpha$ & (iv) Tumor suppression \\
& & (v) Immunostimulation \\
& & (vi) Ability to induce a cytotoxic effect \\
\hline MII & & \\
MIIa & IL-4; IL-13 & (i) Th2 polarization \\
MIIb & Immune complex; & (ii) Down-regulation of adaptive \\
& IL-1R agonists; TLR ligands & immunity \\
MIIc & IL-10; TGF- $\beta$; & (iii) Tumor growth promotion \\
& glucocorticoids & (iv) Proangiogenic \\
(v) Tissue remodeling and repair
\end{tabular}

mannose receptor (MR), and receptor for LPS (CD14) [85].

The principal tasks of MII include the suppression of adaptive response, inhibition of cytotoxic cell activity, rearrangement and reconstruction of destroyed tissues, and their neovascularization. Therefore, MII macrophages play a regulatory function in pro-inflammatory response via the control of MI cell-dependent activities [86]. Consequently, the maintenance of body homeostasis requires maintaining a proper ratio of both discussed subpopulations of macrophages, namely, MI and MII. This balance is disturbed during such pathological conditions as the proliferation of neoplastic cells, leading to the impaired activity of immune system and uncontrolled progression of the disease. This is unambiguously associated with the immunomodulatory effect of proliferating neoplasm. However, the secretory activity of macrophages residing within the tumor should not be forgotten. The activity of macrophages in the course of neoplastic disease has been studied extensively as they can exert both progressive (MII macrophages) and regressive (MI macrophages) effects on the development of neoplastic tissue. The balance between MI and MII macrophages seems to be controlled by $\mathrm{NF} \kappa \mathrm{B}$ signaling, because targeting of this transcription factor switched macrophages from an MII to an MI phenotype. The consequence of which is the regression of tumor tissue in vitro [87].

As pro-inflammatory cells, macrophages are involved in stromal remodeling releasing a slate of pro-inflammatory factors that constitute a signal of danger for immune cells. This macrophage activity may result in the following:

(a) activation of cytotoxic T lymphocytes and NK cells;

(b) influx of dendritic cells;

(c) migration and differentiation of monocytes in a proinflammatory direction.

Activated MI macrophages synthesize and release IL-12, which, as was mentioned previously, shows indirect antineoplastic activity. Additionally, it exerts stimulatory effect on
NK cells, inducing the synthesis and secretion of IFN- $\gamma$ [88] and enhancing their cytotoxic potential. Stimulated with this cytokine, macrophages release factors such as ROI, IL-1, IL6 , arginase, and TNF- $\alpha$, that is, pro-inflammatory factors exerting cytostatic and cytotoxic effects upon neoplastic cells. Moreover, these cells show a strong cytotoxic activity in both the antibody-dependent (ADCC) and antibody-independent (MTC) mechanisms [89].

Unfortunately, the majority of macrophages infiltrating neoplastic tissue have phenotype characteristic for MII [85], and thus they present an array of activities promoting the growth of neoplastic tissue. Consequently, tumor-associated macrophages are considered to constitute MII-like cells. The level of their infiltration is used as an independent prognostic factor in many tumor types. However, it should be noted that in the case of some malignancies, for example, colorectal and gastric cancers, higher fraction of these cells does not necessarily correlate negatively with patients' survival. The activity of TAMs in gastric malignancies can vary depending on tumor region. For example, higher infiltration of TAMs to the region of tumor cell nests is associated with improved survival. Despite the small fraction of nest TAMs, as compared to their overall count in the other regions of the tumor, enhanced apoptosis of neoplastic cells was observed along with a higher activity of cytotoxic $\mathrm{T}$ lymphocytes. Consequently, it should be emphasized that antineoplastic activities controlled by macrophages with MI phenotype can be induced in certain regions of neoplastic tissue [52]. However, they represent a minority of pro-neoplastic activities of MII macrophages, associated with the following:

(a) suppression of adaptive response;

(b) promotion of tumor growth;

(c) promotion of the metastases of neoplastic cells;

(d) involvement in the recruitment of peripheral monocytes and macrophages from the surrounding tissues.

The MII macrophages release an array of anti-inflammatory factors, such as IL-10 and prostanoids, causing the 
attenuation of type Th1 immune response, as well as an impaired activity of cytotoxic T lymphocytes and NK cells. Moreover, they secrete a variety of specific cytokines (e.g., CCL17 and CCL22), inducing the inflow of regulatory $\mathrm{T}$ cells and Th2 subpopulation of helper lymphocytes. These effects are reflected by the suppression of pro-inflammatory activities of the immune system, that is, by the inhibition of activities oriented against neoplastic cells [86]. Moreover, tumor-associated macrophages are capable of modifying $\zeta$ subunit of TCR receptor (TCR- $\zeta$ ) of T-helper lymphocytes [90-92], which plays a crucial role in the activation of the latter cells [93]. The disorders of TCR- $\zeta$ expression or inactivation of this subunit are reflected by the anergy of $\mathrm{T}$ lymphocytes, leading to their apoptosis.

Intensified proliferation of neoplastic tissue is associated with an increased requirement for nutrients and growth factors and leads to the hypoxia of the tumor. High efficiency of pro-neoplastic MII macrophage activity is associated with their ability to accumulate within the oxygendeficient regions of the tissue. TAMs synthesize and release an array of growth and pro-angiogenic factors that are concurrently chemotactic factors for monocytes and macrophages, including VEGF, bFGF, CXCL8, PDGF, EGF, and TGF- $\beta$ $[89,94]$. PDGF promotes the proliferation of neoplastic tissue; additionally, it acts as a pro-angiogenic factor and recruits pericytes stabilizing the newly formed vessels [95]. EGF stimulates neoplastic cells to synthesize and release M-CSF. Aside from the chemotaxis of macrophages from the surrounding tissues, M-CSF induces the differentiation and migration of peripheral monocytes. This constitutes one of the mechanisms behind the enhanced infiltration of macrophages into the tumor, which is in turn reflected by an enhanced synthesis of EGF. EGF/M-CSF feedback cycle leads to macrophage-dependent, growth factor-induced intensive proliferation of neoplastic tissue [96].

Macrophages present at the invasive front of the tumor (margin TAMs) participate in the creation of promoting environment for neoplastic cells, enabling them to reach vascular and lymphatic system. As previously mentioned, TAMs constitute a source of metalloproteinases (such as MMP-2 and MMP-9) and an urokinase-type plasminogen activator (uPA) [97], which facilitate tumor invasion due to their involvement in the degradation of basal membrane and extracellular matrix. This process seems to a large extent to be EGF and M-CSF dependent. The blockade of EGF and MCSF activities is reflected by inhibited migration of neoplastic cells and macrophages, respectively $[98,99]$. Therefore, the M-CSF-stimulated TAMs induce the migration of neoplastic cells on EGF-dependent pathway [100]. Most likely, this process is also modulated by IL-4, which polarizes macrophages towards promoting the invasiveness and spread of the tumor; these functions are blocked in the lack of IL-4 [101, 102]. The invasiveness of neoplastic cells can be also modulated in TNF- $\alpha$-dependent manner [103], with macrophages being the principal source of TNF- $\alpha[104,105]$. The migration of neoplastic cells through the stroma is markedly more efficient if supported by collagen fibers (type I collagen) [106], formed with the involvement of TAMs. These fibers expand towards blood vessels [107], significantly facilitating the migration of neoplastic cells and thus promoting the formation of distant metastases. In summary, due to their pro-angiogenic activity, synthesis of collagen fibers, and the induction of neoplastic cell migration, TAMs actively promote the invasion and spread of the tumor.

\section{Conclusion}

The process of neoplastic tissue proliferation is directly related to the modulatory effect on the immune system. Monocytes/macrophages are particularly susceptible to this effect. This results in a switch from a protective function into one that promotes neoplastic proliferation. In the case of many malignancies (e.g., breast, prostate, or endometrial cancer), high percentage of TAMs is associated with poor prognosis. In practice, high percentage of tumor-associated macrophages may be an independent prognostic factor, providing a thorough examination of all the regions of the tumor and a precise assessment of the phenotype of these cells. TAMs also seem to be a promising target for antineoplastic therapy, the aim of which should be, for example, the reversal of the unfavorable balance between MI and MII macrophages [87]. On the other hand, monocytes/macrophages can be used as a delivery system of anticancer agents to tumors [108].

\section{Acknowledgment}

This paper was supported by the "Grant for statutory activities" no. 837 from Collegium Medicum in Bydgoszcz, Nicolaus Copernicus University of Torun.

\section{References}

[1] E. C. Friedberg, “DNA damage and repair," Nature, vol. 421, no. 6921, pp. 436-440, 2003.

[2] C. Bernstein, H. Bernstein, C. M. Payne, and H. Garewal, "DNA repair/pro-apoptotic dual-role proteins in five major DNA repair pathways: fail-safe protection against carcinogenesis," Mutation Research, vol. 511, no. 2, pp. 145-178, 2002.

[3] E. R. Fearon and B. Vogelstein, "A genetic model for colorectal tumorigenesis," Cell, vol. 61, no. 5, pp. 759-767, 1990.

[4] T. Tanaka, Z. Bai, Y. Srinoulprasert, B. Yang, H. Hayasaka, and M. Miyasaka, "Chemokines in tumor progression and metastasis," Cancer Science, vol. 96, no. 6, pp. 317-322, 2005.

[5] A. D. Luster, "Chemokines-chemotactic cytokines that mediate inflammation," The New England Journal of Medicine, vol. 338, no. 7, pp. 436-445, 1998.

[6] B. Bottazzi, F. Colotta, A. Sica, N. Nobili, and A. Mantovani, "A chemoattractant expressed in human sarcoma cells (tumorderived chemotactic factor, TDCF) is identical to monocyte chemoattractant protein-1/monocyte chemotactic and activating factor (MCP-1/MCAF)," International Journal of Cancer, vol. 45, no. 4, pp. 795-797, 1990.

[7] I. Conti and B. J. Rollins, "CCL2 (monocyte chemoattractant protein-1) and cancer," Seminars in Cancer Biology, vol. 14, no. 3, pp. 149-154, 2004.

[8] B. Amann, F. G. E. Perabo, A. Wirger, H. Hugenschmidt, and W. Schultze-Seemann, "Urinary levels of monocyte chemoattractant protein-1 correlate with tumour stage and grade in 
patients with bladder cancer," British Journal of Urology, vol. 82, no. 1, pp. 118-121, 1998.

[9] T. Valković, K. Lučin, M. Krstulja, R. Dobi-Babić, and N. Jonjić, "Expression of monocyte chemotactic protein-1 in human invasive ductal breast cancer," Pathology Research and Practice, vol. 194, no. 5, pp. 335-340, 1998.

[10] H. Tonouchi, C. Miki, K. Tanaka, and M. Kusunoki, "Profile of monocyte chemoattractant protein-1 circulating levels in gastric cancer patients," Scandinavian Journal of Gastroenterology, vol. 37, no. 7, pp. 830-833, 2002.

[11] T. Ueno, M. Toi, H. Saji et al., "Significance of macrophage chemoattractant protein-1 in macrophage recruitment, angiogenesis, and survival in human breast cancer," Clinical Cancer Research, vol. 6, no. 8, pp. 3282-3289, 2000.

[12] A. Sica, A. Saccani, B. Bottazzi et al., "Defective expression of the monocyte chemotactic protein-1 receptor CCR2 in macrophages associated with human ovarian carcinoma," Journal of Immunology, vol. 164, no. 2, pp. 733-738, 2000.

[13] J. Van Damme, P. Proost, J. P. Lenaerts, and G. Opdenakker, "Structural and functional identification of two human, tumorderived monocyte chemotactic proteins (MCP-2 and MCP-3) belonging to the chemokine family," Journal of Experimental Medicine, vol. 176, no. 1, pp. 59-65, 1992.

[14] G. Luboshits, S. Shina, O. Kaplan et al., "Elevated expression of the CC chemokine regulated on activation, normal $\mathrm{T}$ cell expressed and secreted (RANTES) in advanced breast carcinoma," Cancer Research, vol. 59, no. 18, pp. 4681-4687, 1999.

[15] M. Locati, U. Deuschle, M. L. Massardi et al., "Analysis of the gene expression profile activated by the CC chemokine ligand 5/RANTES and by lipopolysaccharide in human monocytes," Journal of Immunology, vol. 168, no. 7, pp. 3557-3562, 2002.

[16] S. C. Robinson, K. A. Scott, and F. R. Balkwill, "Chemokine stimulation of monocyte matrix metalloproteinase-9 requires endogenous TNF-alpha," European Journal of Immunology, vol. 32, pp. 404-412, 2002.

[17] H. K. Kim, K. S. Song, Y. S. Park et al., "Elevated levels of circulating platelet microparticles, VEGF, IL-6 and RANTES in patients with gastric cancer: possible role of a metastasis predictor," European Journal of Cancer, vol. 39, pp. 184-191, 2003.

[18] J. Folkman, "Angiogenesis in cancer, vascular, rheumatoid and other disease," Nature Medicine, vol. 1, no. 1, pp. 27-31, 1995.

[19] J. Folkman, K. Watson, D. Ingber, and D. Hanahan, "Induction of angiogenesis during the transition from hyperplasia to neoplasia," Nature, vol. 339, no. 6219, pp. 58-61, 1989.

[20] J. Folkman, "What is the evidence that tumors are angiogenesis dependent?" Journal of the National Cancer Institute, vol. 82, no. 1, pp. 4-6, 1990.

[21] S. Kido, Y. Kitadai, N. Hattori et al., "Interleukin 8 and vascular endothelial growth factor-prognostic factors in human gastric carcinomas?" European Journal of Cancer, vol. 37, no. 12, pp. 1482-1487, 2001.

[22] M. Toi, S. Kondo, H. Suzuki et al., "Quantitative analysis of vascular endothelial growth factor in primary breast cancer," Cancer, vol. 77, pp. 1101-1106, 1996.

[23] K. Fujisaki, K. Mitsuyama, A. Toyonaga, K. Matsuo, and K. Tanikawa, "Circulating vascular endothelial growth factor in patients with colorectal cancer," American Journal of Gastroenterology, vol. 93, no. 2, pp. 249-252, 1998.

[24] S. Kiriakidis, E. Andreakos, C. Monaco, B. Foxwell, M. Feldmann, and E. Paleolog, "VEGF expression in human macrophages is NF- $\kappa \mathrm{B}$-dependent: studies using adenoviruses expressing the endogenous NF- $\kappa \mathrm{B}$ inhibitor $\mathrm{I} \kappa \mathrm{B} \alpha$ and a kinasedefective form of the I $\kappa$ B kinase 2," Journal of Cell Science, vol. 116, no. 4, pp. 665-674, 2003.

[25] M. Shibuya, "Structure and function of VEGF/VEGF-receptor system involved in angiogenesis," Cell Structure and Function, vol. 26, no. 1, pp. 25-35, 2001.

[26] A. Sawano, S. Iwai, Y. Sakurai et al., "Flt-1, vascular endothelial growth factor receptor 1 , is a novel cell surface marker for the lineage of monocyte-macrophages in humans," Blood, vol. 97, no. 3, pp. 785-791, 2001.

[27] M. Hollborn, C. Stathopoulos, A. Steffen, P. Wiedemann, L. Kohen, and A. Bringmann, "Positive feedback regulation between MMP-9 and VEGF in human RPE cells," Investigative Ophthalmology and Visual Science, vol. 48, no. 9, pp. 4360-4367, 2007.

[28] S. Hiratsuka, K. Nakamura, S. Iwai et al., "MMP9 induction by vascular endothelial growth factor receptor-1 is involved in lung-specific metastasis," Cancer Cell, vol. 2, no. 4, pp. 289-300, 2002.

[29] L. Bingle, N. J. Brown, and C. E. Lewis, "The role of tumourassociated macrophages in tumour progression: implications for new anticancer therapies," Journal of Pathology, vol. 196, no. 3, pp. 254-265, 2002.

[30] H. F. Dvorak, L. F. Brown, M. Detmar, and A. M. Dvorak, "Vascular permeability factor/vascular endothelial growth factor, microvascular hyperpermeability, and angiogenesis," American Journal of Pathology, vol. 146, no. 5, pp. 1029-1039, 1995.

[31] G. Neufeld, T. Cohen, S. Gengrinovitch, and Z. Poltorak, "Vascular endothelial growth factor (VEGF) and its receptors," The FASEB Journal, vol. 13, no. 1, pp. 9-22, 1999.

[32] P. Lissoni, F. Malugani, A. Bonfanti et al., "Abnormally enhanced blood concentrations of vascular endothelial growth factor (VEGF) in metastatic cancer patients and their relation to circulating dendritic cells, IL-12 and endothelin-1," Journal of Biological Regulators and Homeostatic Agents, vol. 15, no. 2, pp. 140-144, 2001.

[33] N. Ferrara, K. J. Hillan, H. P. Gerber, and W. Novotny, "Discovery and development of bevacizumab, an anti-VEGF antibody for treating cancer," Nature Reviews Drug Discovery, vol. 3, no. 5, pp. 391-400, 2004.

[34] Y. Kitadai, "Angiogenesis and lymphangiogenesis of gastric cancer," Journal of Oncology, vol. 2010, Article ID 468725, 8 pages, 2010.

[35] K. Norrby, "Basic fibroblast growth factor and de novo mammalian angiogenesis," Microvascular Research, vol. 48, no. 1, pp. 96-113, 1994.

[36] I. Bilgic, N. Ozalp, M. Tez, and M. Koc, "Serum bFGF concentrations in gastric cancer patients," Bratislavské Lekárske Listy, vol. 109, no. 1, pp. 8-9, 2008.

[37] I. Esposito, M. Menicagli, N. Funel et al., "Inflammatory cells contribute to the generation of an angiogenic phenotype in pancreatic ductal adenocarcinoma," Journal of Clinical Pathology, vol. 57, no. 6, pp. 630-636, 2004.

[38] C. E. Lewis, R. Leek, A. Harris, and O. J. McGee, "Cytokine regulation of angiogenesis in breast cancer: the role of tumorassociated macrophages," Journal of Leukocyte Biology, vol. 57, no. 5, pp. 747-751, 1995.

[39] M. J. Jo, J. H. Lee, B. H. Nam et al., "Preoperative serum angiopoietin-2 levels correlate with lymph node status in patients with early gastric cancer," Annals of Surgical Oncology, vol. 16, no. 7, pp. 2052-2057, 2009. 
[40] J. Wang, K. Wu, D. Zhang et al., "Expressions and clinical significances of angiopoietin-1, -2 and Tie2 in human gastric cancer," Biochemical and Biophysical Research Communications, vol. 337, no. 1, pp. 386-393, 2005.

[41] M. Hangai, T. Murata, N. Miyawaki et al., "Angiopoietin-1 upregulation by vascular endothelial growth factor in human retinal pigment epithelial cells," Investigative Ophthalmology and Visual Science, vol. 42, no. 7, pp. 1617-1625, 2001.

[42] S. Niedźwiecki, T. Stepień, K. Kopeć et al., "Angiopoietin 1 (Ang1), angiopoietin 2 (Ang-2) and Tie-2 (a receptor tyrosine kinase) concentrations in peripheral blood of patients with thyroid cancers," Cytokine, vol. 36, no. 5-6, pp. 291-295, 2006.

[43] C. Murdoch, S. Tazzyman, S. Webster, and C. E. Lewis, "Expression of Tie- 2 by human monocytes and their responses to angiopoietin-2," Journal of Immunology, vol. 178, no. 11, pp. 7405-7411, 2007.

[44] A. Szkaradkiewicz, T. M. Karpiński, M. Drews, M. BorejszaWysocki, P. Majewski, and E. Andrzejewska, "Natural killer cell cytotoxicity and immunosuppressive cytokines (IL-10, TGF$\beta 1$ ) in patients with gastric cancer," Journal of Biomedicine and Biotechnology, vol. 2010, Article ID 901564, 7 pages, 2010.

[45] H. Groux, M. Bigler, J. E. De Vries, and M. G. Roncarolo, "Inhibitory and stimulatory effects of IL-10 on Human CD8+ T cells," Journal of Immunology, vol. 160, no. 7, pp. 3188-3193, 1998.

[46] S. Yano, S. Sone, Y. Nishioka, N. Mukaida, K. Matsushima, and T. Ogura, "Differential effects of anti-inflammatory cytokines (IL-4, IL-10 and IL-13) on tumoricidal and chemotactic properties of human monocytes induced by monocyte chemotactic and activating factor," Journal of Leukocyte Biology, vol. 57, no. 2, pp. 303-309, 1995.

[47] J. C. Becker, C. Czerny, and E. B. Brocker, "Maintenance of clonal anergy by endogenously produced IL-10," International Immunology, vol. 6, no. 10, pp. 1605-1612, 1994.

[48] R. Kim, M. Emi, K. Tanabe, and K. Arihiro, "Tumor-driven evolution of immunosuppressive networks during malignant progression," Cancer Research, vol. 66, no. 11, pp. 5527-5536, 2006.

[49] S. Chouaib, C. Asselin-Paturel, F. Mami-Chouaib, A. Caignard, and J. Y. Blay, "The host-tumor immune conflict: from immunosuppression to resistance and destruction," Immunology Today, vol. 18, no. 10, pp. 493-497, 1997.

[50] C. Chen, Y. Shen, Q. X. Qu, X. Q. Chen, X. G. Zhang, and J. A. Huang, "Induced expression of B7-H3 on the lung cancer cells and macrophages suppresses T-cell mediating anti-tumor immune response," Experimental Cell Research, vol. 319, no. 1, pp. 96-102, 2013.

[51] S. Gordon and P. R. Taylor, "Monocyte and macrophage heterogeneity," Nature Reviews Immunology, vol. 5, no. 12, pp. 953-964, 2005.

[52] S. Ohno, H. Inagawa, D. K. Dhar et al., "The degree of macrophage infiltration into the cancer cell nest is a significant predictor of survival in gastric cancer patients," Anticancer Research, vol. 23, no. 6, pp. 5015-5022, 2003.

[53] R. D. Leek, C. E. Lewis, R. Whitehouse, M. Greenall, J. Clarke, and A. L. Harris, "Association of macrophage infiltration with angiogenesis and prognosis in invasive breast carcinoma," Cancer Research, vol. 56, no. 20, pp. 4625-4629, 1996.

[54] S. Shimura, G. Yang, S. Ebara, T. M. Wheeler, A. Frolov, and T. C. Thompson, "Reduced infiltration of tumor-associated macrophages in human prostate cancer: association with cancer progression," Cancer Research, vol. 60, no. 20, pp. 5857-5861, 2000.

[55] D. J. T. Maliszewski, K. Drucis, and A. Kopacz, "Czynniki prognostyczne w raku jelita grubego-co możemy dodaćdo standardu?” Współczesna Onkologia, vol. 12, pp. 212-216, 2008.

[56] L. Ziegler-Heitbrock, P. Ancuta, S. Crowe et al., "Nomenclature of monocytes and dendritic cells in blood," Blood, vol. 116, no. 16, pp. e74-e80, 2010.

[57] H. W. L. Ziegler-Heitbrock, "Heterogeneity of human blood monocytes: the CD14+CD16+ subpopulation," Immunology Today, vol. 17, no. 9, pp. 424-428, 1996.

[58] E. Grage-Griebenow, H. D. Flad, and M. Ernst, "Heterogeneity of human peripheral blood monocyte subsets," Journal of Leukocyte Biology, vol. 69, no. 1, pp. 11-20, 2001.

[59] J. Skrzeczyńska-Moncznik, M. Bzowska, S. Loseke, E. GrageGriebenow, M. Zembala, and J. Pryjma, "Peripheral blood CD14high CD16+ monocytes are main producers of IL-10," Scandinavian Journal of Immunology, vol. 67, no. 2, pp. 152-159, 2008.

[60] W. A. Nockher and J. E. Scherberich, "Expanded CD14+ CD16+ Monocyte subpopulation in patients with acute and chronic infections undergoing hemodialysis," Infection and Immunity, vol. 66, no. 6, pp. 2782-2790, 1998.

[61] K. Katayama, T. Matsubara, M. Fujiwara, M. Koga, and S. Furukawa, "CD14+CD16+ monocyte subpopulation in Kawasaki disease," Clinical and Experimental Immunology, vol. 121, no. 3, pp. 566-570, 2000.

[62] G. Fingerle, A. Pforte, B. Passlick, M. Blumenstein, M. Strobel, and H. W. L. Ziegler- Heitbrock, "The novel subset of CD14+/CD16+ blood monocytes is expanded in sepsis patients," Blood, vol. 82, no. 10, pp. 3170-3176, 1993.

[63] A. L. Feng, J. K. Zhu, J. T. Sun et al., "CD16+ monocytes in breast cancer patients: expanded by monocyte chemoattractant protein-1 and may be useful for early diagnosis," Clinical and Experimental Immunology, vol. 164, no. 1, pp. 57-65, 2011.

[64] M. N. Saleh, S. J. Goldman, A. F. LoBuglio et al., "CD16+ monocytes in patients with cancer: spontaneous elevation and pharmacologic induction by recombinant human macrophage colony-stimulating factor," Blood, vol. 85, no. 10, pp. 2910-2917, 1995.

[65] K. U. Belge, F. Dayyani, A. Horelt et al., “The proinflammatory CD14+CD16+DR++ monocytes are a major source of TNF," Journal of Immunology, vol. 168, no. 7, pp. 3536-3542, 2002.

[66] L. Ziegler-Heitbrock, "The CD14+ CD16+ blood monocytes: their role in infection and inflammation," Journal of Leukocyte Biology, vol. 81, no. 3, pp. 584-592, 2007.

[67] A. Rivier, J. Pene, H. Rabesandratana, P. Chanez, J. Bousquet, and A. M. Campbell, "Blood monocytes of untreated asthmatics exhibit some features of tissue macrophages," Clinical and Experimental Immunology, vol. 100, no. 2, pp. 314-318, 1995.

[68] J. Skrzeczyñska, K. Kobylarz, Z. Hartwich, M. Zembala, and J. Pryjma, "CD14+CD16+ monocytes in the course of sepsis in neonates and small children: monitoring and functional studies," Scandinavian Journal of Immunology, vol. 55, no. 6, pp. 629-638, 2002.

[69] A. Eljaszewicz, M. Jankowski, L. Gackowska et al., "Gastric cancer increase the percentage of intermediate (CD14++CD16+) and nonclassical (CD14+CD16+) monocytes," Central European Journal of Immunology, vol. 37, pp. 355-361, 2012.

[70] G. Rothe, H. Gabriel, E. Kovacs et al., "Peripheral blood mononuclear phagocyte subpopulations as cellular markers in 
hypercholesterolemia," Arteriosclerosis, Thrombosis, and Vascular Biology, vol. 16, no. 12, pp. 1437-1447, 1996.

[71] A. Szaflarska, M. Baj-Krzyworzeka, M. Siedlar et al., "Antitumor response of CD14+/CD16+ monocyte subpopulation," Experimental Hematology, vol. 32, no. 8, pp. 748-755, 2004.

[72] M. P. Colombo and G. Trinchieri, "Interleukin-12 in anti-tumor immunity and immunotherapy," Cytokine and Growth Factor Reviews, vol. 13, no. 2, pp. 155-168, 2002.

[73] S. J. Ha, C. H. Lee, S. B. Lee et al., "A novel function of IL12 p40 as a chemotactic molecule for macrophages," Journal of Immunology, vol. 163, no. 5, pp. 2902-2908, 1999.

[74] H. Nakayama, J. Kitayama, T. Muto, and H. Nagawa, "Characterization of intracellular cytokine profile of CD4(+) T cells in peripheral blood and tumor-draining lymph nodes of patients with gastrointestinal cancer," Japanese Journal of Clinical Oncology, vol. 30, no. 7, pp. 301-305, 2000.

[75] H. G. Augustin, G. Young Koh, G. Thurston, and K. Alitalo, "Control of vascular morphogenesis and homeostasis through the angiopoietin-tie system," Nature Reviews Molecular Cell Biology, vol. 10, no. 3, pp. 165-177, 2009.

[76] M. A. Venneri, M. De Palma, M. Ponzoni et al., "Identification of proangiogenic TIE2-expressing monocytes (TEMs) in human peripheral blood and cancer," Blood, vol. 109, no. 12, pp. 52765285, 2007.

[77] M. De Palma, M. A. Venneri, C. Roca, and L. Naldini, "Targeting exogenous genes to tumor angiogenesis by transplantation of genetically modified hematopoietic stem cells," Nature Medicine, vol. 9, no. 6, pp. 789-795, 2003.

[78] M. Benoit, E. Ghigo, C. Capo, D. Raoult, and J. L. Mege, “The uptake of apoptotic cells drives Coxiella burnetii replication and macrophage polarization: a model for Q fever endocarditis," PLoS Pathogens, vol. 4, no. 5, Article ID e1000066, 2008.

[79] A. Mantovani, A. Sica, S. Sozzani, P. Allavena, A. Vecchi, and M. Locati, "The chemokine system in diverse forms of macrophage activation and polarization," Trends in Immunology, vol. 25, no. 12, pp. 677-686, 2004.

[80] C. M. Ohri, A. Shikotra, R. H. Green, D. A. Waller, and P. Bradding, "Macrophages within NSCLC tumour islets are predominantly of a cytotoxic M1 phenotype associated with extended survival," European Respiratory Journal, vol. 33, no. 1, pp. 118-126, 2009.

[81] Y. Ohmori and T. A. Hamilton, "Requirement for STAT1 in LPSinduced gene expression in macrophages," Journal of Leukocyte Biology, vol. 69, no. 4, pp. 598-604, 2001.

[82] S. Akira, “Toll-like receptor signaling," Journal of Biological Chemistry, vol. 278, no. 40, pp. 38105-38108, 2003.

[83] S. Ito, P. Ansari, M. Sakatsume et al., "Interleukin-10 inhibits expression of both interferon $\alpha$ - and interferon $\gamma$-induced genes by suppressing tyrosine phosphorylation of STAT1," Blood, vol. 93, no. 5, pp. 1456-1463, 1999.

[84] S. B. Coffelt, R. Hughes, and C. E. Lewis, "Tumor-associated macrophages: effectors of angiogenesis and tumor progression," Biochimica et Biophysica Acta, vol. 1796, no. 1, pp. 11-18, 2009.

[85] A. Mantovani, S. Sozzani, M. Locati, P. Allavena, and A. Sica, "Macrophage polarization: tumor-associated macrophages as a paradigm for polarized M2 mononuclear phagocytes," Trends in Immunology, vol. 23, no. 11, pp. 549-555, 2002.

[86] G. Solinas, G. Germano, A. Mantovani, and P. Allavena, "Tumor-associated macrophages (TAM) as major players of the cancer-related inflammation," Journal of Leukocyte Biology, vol. 86, no. 5, pp. 1065-1073, 2009.
[87] T. Hagemann, T. Lawrence, I. McNeish et al., "Re-educating' tumor-associated macrophages by targeting NF- $\kappa \mathrm{B}$," Journal of Experimental Medicine, vol. 205, no. 6, pp. 1261-1268, 2008.

[88] T. Germann and E. Rude, “Interleukin-12," International Archives of Allergy and Immunology, vol. 108, no. 2, pp. 103-112, 1995.

[89] B. Al-Sarireh and O. Eremin, "Tumour-associated macrophages (TAMS): disordered function, immune suppression and progressive tumour growth," Journal of the Royal College of Surgeons of Edinburgh, vol. 45, no. 1, pp. 1-16, 2000.

[90] J. Sikora, G. Dworacki, R. Giersz, and J. Zeromski, “The role of monocytes/macrophages in TCR- $\zeta$ chain downregulation and apoptosis of T lymphocytes in malignant pleural effusions," Journal of Biological Regulators and Homeostatic Agents, vol. 18, no. 1, pp. 26-32, 2004.

[91] S. J. Guo, D. M. Lin, J. Li et al., “Tumor-associated macrophages and $\mathrm{CD} 3-\zeta$ expression of tumor-infiltrating lymphocytes in human esophageal squamous-cell carcinoma," Diseases of the Esophagus, vol. 20, no. 2, pp. 107-116, 2007.

[92] W. M. C. Mulder, E. Bloemena, M. J. Stukart, J. A. Kummer, J. Wagstaff, and R. J. Scheper, "T cell receptor- $\zeta$ and granzyme $\mathrm{B}$ expression in mononuclear cell infiltrates in normal colon mucosa and colon carcinoma," Gut, vol. 40, no. 1, pp. 113-119, 1997.

[93] E. N. Kersh, A. S. Shaw, and P. M. Allen, "Fidelity of T cell activation through multistep T cell receptor $\zeta$ phosphorylation," Science, vol. 281, no. 5376, pp. 572-575, 1998.

[94] S. K. Leivonen and V. M. Kähäri, "Transforming growth factor$\beta$ signaling in cancer invasion and metastasis," International Journal of Cancer, vol. 121, no. 10, pp. 2119-2124, 2007.

[95] C. Lamagna, M. Aurrand-Lions, and B. A. Imhof, "Dual role of macrophages in tumor growth and angiogenesis," Journal of Leukocyte Biology, vol. 80, no. 4, pp. 705-713, 2006.

[96] J.-Y. Shih, A. Yuan, J. J. W. Chen, and P. C. Yang, "Tumorassociated macrophage: its role in cancer invasion and metastasis," Journal of Cancer Molecules, vol. 2, pp. 101-106, 2006.

[97] R. Hildenbrand, G. Wolf, B. Böhme, U. Bleyl, and A. Steinborn, "Urokinase plasminogen activator receptor (CD87) expression of tumor- associated macrophages in ductal carcinoma in situ, breast cancer, and resident macrophages of normal breast tissue," Journal of Leukocyte Biology, vol. 66, no. 1, pp. 40-49, 1999.

[98] E. Y. Lin, A. V. Nguyen, R. G. Russell, and J. W. Pollard, “Colonystimulating factor 1 promotes progression of mammary tumors to malignancy," Journal of Experimental Medicine, vol. 193, no. 6, pp. 727-739, 2001.

[99] J. Wyckoff, W. Wang, E. Y. Lin et al., "A paracrine loop between tumor cells and macrophages is required for tumor cell migration in mammary tumors," Cancer Research, vol. 64, no. 19, pp. 7022-7029, 2004.

[100] S. Goswami, E. Sahai, J. B. Wyckoff et al., "Macrophages promote the invasion of breast carcinoma cells via a colonystimulating factor-1/epidermal growth factor paracrine loop," Cancer Research, vol. 65, pp. 5278-5283, 2005.

[101] D. G. DeNardo, J. B. Barreto, P. Andreu et al., "CD4+ T cells regulate pulmonary metastasis of mammary carcinomas by enhancing protumor properties of macrophages," Cancer Cell, vol. 16, no. 2, pp. 91-102, 2009.

[102] V. Gocheva, H. W. Wang, B. B. Gadea et al., "IL-4 induces cathepsin protease activity in tumor-associated macrophages to promote cancer growth and invasion," Genes and Development, vol. 24, no. 3, pp. 241-255, 2010. 
[103] T. Hagemann, J. Wilson, H. Kulbe et al., "Macrophages induce invasiveness of epithelial cancer cells via NF- $\kappa$ B and JNK," Journal of Immunology, vol. 175, no. 2, pp. 1197-1205, 2005.

[104] Q. Li and I. M. Verma, "NF-kappaB regulation in the immune system," Nature Reviews Immunology, vol. 2, pp. 725-734, 2002.

[105] G. Bonizzi and M. Karin, "The two NF- $\kappa$ B activation pathways and their role in innate and adaptive immunity," Trends in Immunology, vol. 25, no. 6, pp. 280-288, 2004.

[106] J. B. Wyckoff, Y. Wang, E. Y. Lin et al., "Direct visualization of macrophage-assisted tumor cell intravasation in mammary tumors," Cancer Research, vol. 67, no. 6, pp. 2649-2656, 2007.

[107] W. V. Ingman, J. Wyckoff, V. Gouon-Evans, J. Condeelis, and J. W. Pollard, "Macrophages promote collagen fibrillogenesis around terminal end buds of the developing mammary gland," Developmental Dynamics, vol. 235, no. 12, pp. 3222-3229, 2006.

[108] J. Choi, H. Y. Kim, E. J. Ju et al., "Use of macrophages to deliver therapeutic and imaging contrast agents to tumors," Biomaterials, vol. 33, pp. 4195-4203, 2012. 


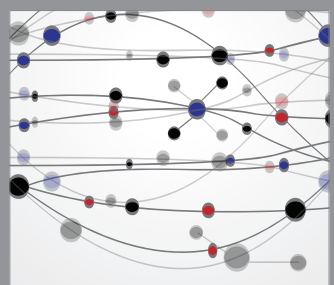

The Scientific World Journal
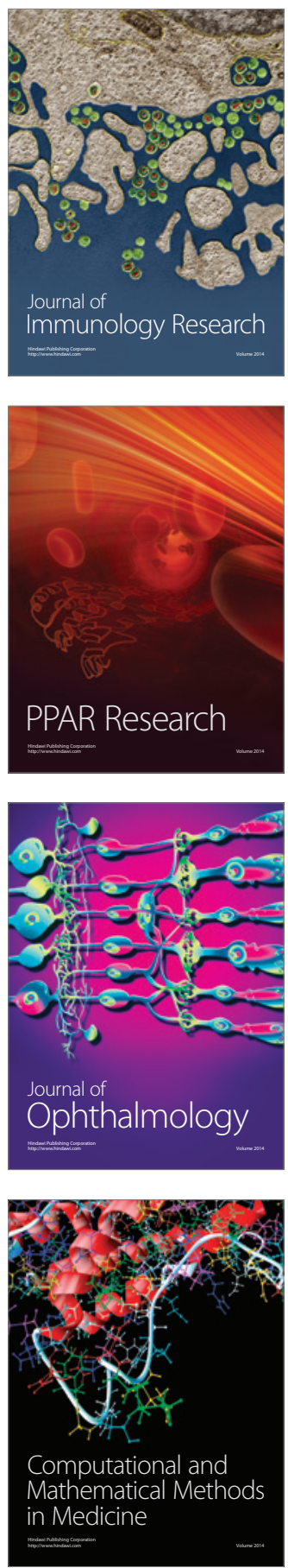

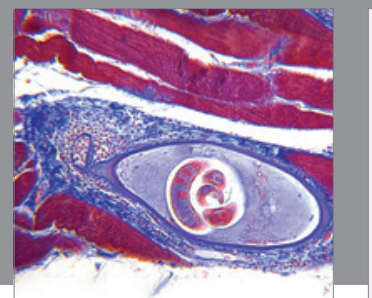

Gastroenterology

Research and Practice
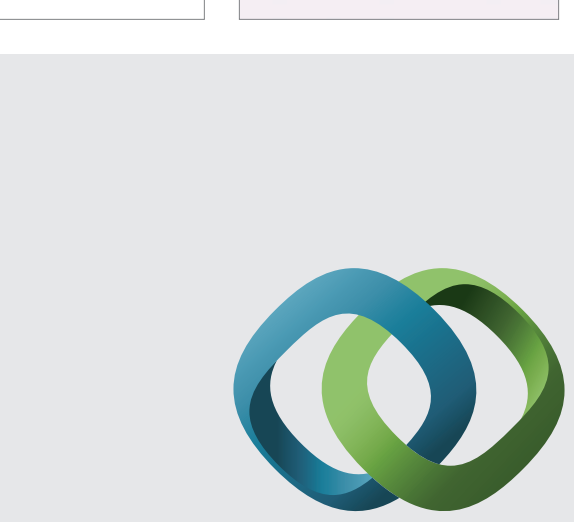

\section{Hindawi}

Submit your manuscripts at

http://www.hindawi.com
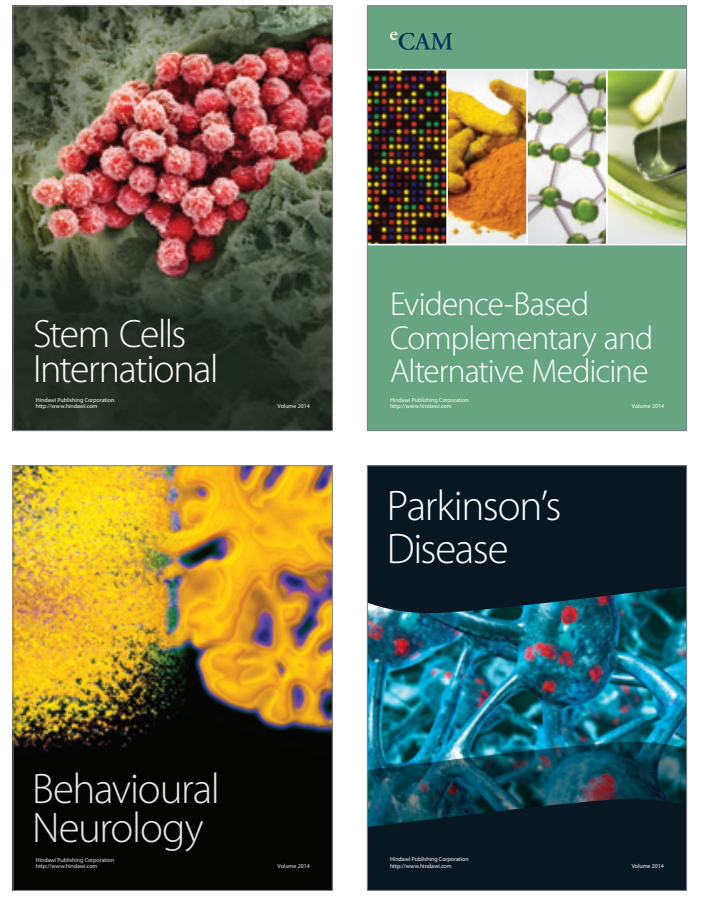
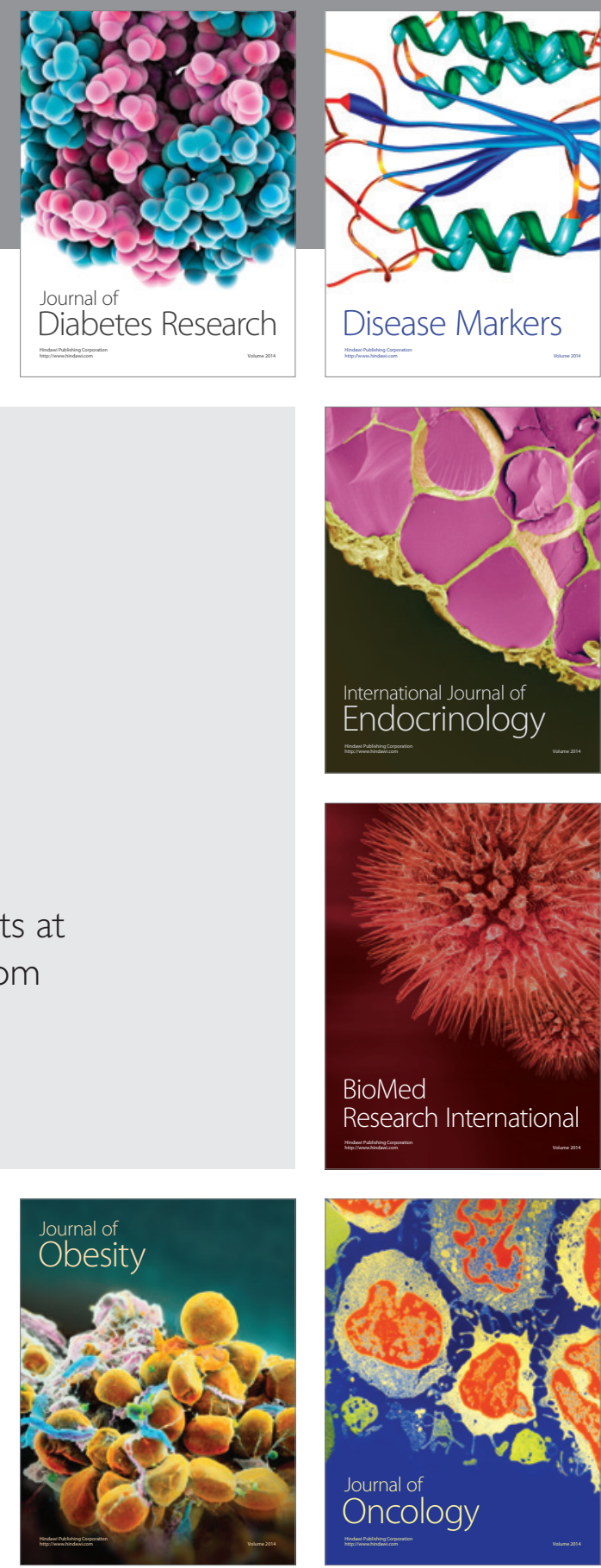

Disease Markers
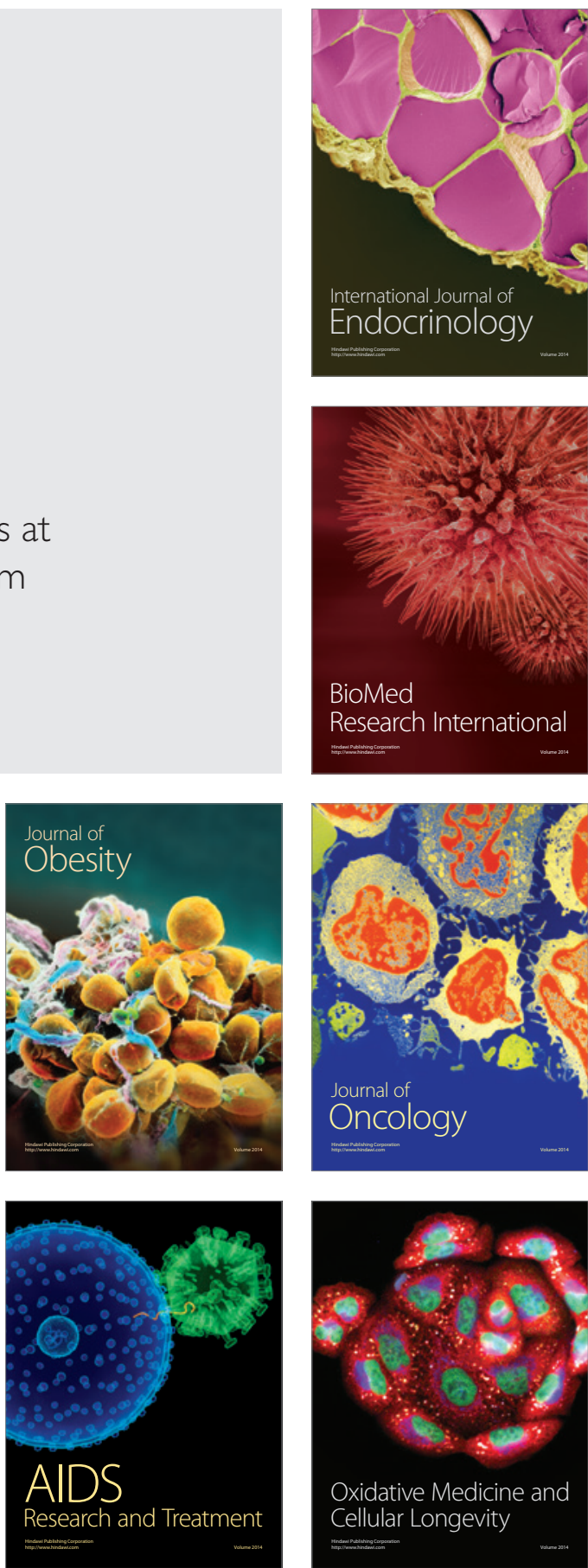